\title{
ERS International Congress, Madrid, 2019: highlights from the Sleep and Clinical Physiology Assembly
}

\begin{abstract}
Andrea Crespo $\mathbb{1}^{1,2,12}$, Sébastien Baillieul $\mathbb{1}^{3,4,12}$, Esther Marhuenda ${ }^{5,6,12}$, Matteo Bradicich ${ }^{7,12}$, 'Vasileios Andrianopoulos (10, 8,12 , Zafeiris Louvaris ${ }^{9,12}$, Mathieu Marillier ${ }^{10,12}$ and Isaac Almendros $\mathbb{1}^{5,6,11,12^{\prime}}$
\end{abstract}

Affiliations: ${ }^{1}$ Multidisciplinary Sleep Unit, Dept of Pulmonology, Rio Hortega University Hospital, Valladolid, Spain. ${ }^{2}$ Biomedical Engineering Group, University of Valladolid, Valladolid, Spain. ${ }^{3} \mathrm{HP} 2$ Laboratory, INSERM U1042, Grenoble Alpes University, Grenoble, France. ${ }^{4}$ FCR Laboratory, Grenoble Alpes University Hospital, Grenoble, France. ${ }^{5}$ Unitat de Biofísica i Bioenginyeria, Facultat de Medicina i Ciències de la Salut, Universitat de Barcelona, Barcelona, Spain. ${ }^{6}$ Centro de Investigación Biomédica en Red de Enfermedades Respiratorias CIBERES, Madrid, Spain. ${ }^{7}$ Dept of Pulmonology and Sleep Disorders Centre, University Hospital Zurich, Zurich, Switzerland. ${ }^{8}$ Institute for Pulmonary Rehabilitation Research, Schoen Klinik Berchtesgadener Land, Schoenau am Koenigssee, Germany. ${ }^{9}$ Faculty of Kinesiology and Rehabilitation Sciences, Division of Respiratory Rehabilitation, Department Rehabilitation Sciences KU Leuven, University Hospitals Leuven, Leuven, Belgium. ${ }^{10}$ Laboratory of Clinical Exercise Physiology, Queen's University, Kingston, ON, Canada. ${ }^{11}$ Institut d'Investigacions Biomèdiques August Pi i Sunyer (IDIBAPS), Barcelona, Spain. ${ }^{12}$ All authors contributed equally to this work.

Correspondence: Andrea Crespo, Multidisciplinary Sleep Unit, Dept of Pulmonary Medicine, Rio Hortega University Hospital, Dulzaina 2, 47012, Valladolid, Spain. E-mail: acresposasaludcastillayleon.es/

ABSTRACT The 2019 European Respiratory Society (ERS) International Congress took place in Madrid, Spain, and served as a platform to find out the latest advances in respiratory diseases research. The research aims are to understand the physiology and consequences of those diseases, as well as the improvement in their diagnoses, treatments and patient care. In particular, the scientific sessions arranged by ERS Assembly 4 provided novel insights into sleep-disordered breathing and new knowledge in respiratory physiology. This article, divided by session, will summarise the most relevant studies presented at the ERS International Congress. Each section has been written by Early Career Members specialising in the different fields of this interdisciplinary assembly.

@ERSpublications

A selection of the best sessions from @ERS_assembly4 at the 2019 \#ERSCongress by Early Career @EuroRespSoc members https://bit.ly/3g30X6W

Cite this article as: Crespo A, Baillieul S, Marhuenda E, et al. ERS International Congress, Madrid, 2019: highlights from the Sleep and Clinical Physiology Assembly. ERJ Open Res 2020; 6: 00373-2019 [https://doi.org/10.1183/23120541.00373-2019].

Received: 28 Dec 2019 | Accepted after revision: 13 May 2020 | First published: 20 July 2020

Copyright $\odot$ ERS 2020. This article is open access and distributed under the terms of the Creative Commons Attribution Non-Commercial Licence 4.0. 


\section{State of the art session: sleep and breathing disorders}

This symposium was devoted to the advances in the field of sleep and breathing disorders encompassing a broad range of conditions, both in children and adults, with special attention to the underlying pathophysiological mechanisms.

Refika Hamutcu (Ottawa, Canada) talked about intermittent hypoxia and sleep disorders in children. Paediatric obstructive sleep apnoea syndrome (OSAS) is a common health problem, recently highlighted in two European Respiratory Society (ERS) statements [1,2]. Paediatric OSAS, if untreated, may result in significant cardiovascular [1] and metabolic morbidity and growth retardation, as well as neurocognitive, depressive [3] and behavioural consequences that dampen academic performance [4]. Evidence supports that intermittent hypoxia plays an important role in the pathophysiology of sleep-disordered breathing (SDB) morbidity in children. However, for neurocognitive morbidity, it is still unclear which factors play the most important roles. In a study by KHEIRANDISH-Gozal et al. [5], the respiratory arousal index was a significant predictor of both learning slope and morning recall function, even after adjusting for age, sex, ethnicity, maximal individual performance and total sleep duration [5]. From a therapeutic point of view, cognition wasn't improved 7 months after adenotonsillectomy [6]. However, the surgical treatment of SDB has long-term effects, as improvements in some areas of neurocognition were observed 4 years after the treatment [7]. Interestingly, 3 months of continuous positive airway pressure (CPAP) therapy was associated with improvements of neuro-behavioural outcomes in children with OSAS [8]. Altogether, these results reflect the complexity of the relationship between OSAS and neural deficits, highlighting the need for further studies in this domain. Due to the multisystemic adverse effects of paediatrics OSAS, parents, teachers and clinicians must be able to recognise children with this disease [9].

Marisa Bonsignore (Palermo, Italy) provided an overview of the relationships between asthma, obesity, metabolic dysfunction and OSAS. Obesity increases the risk for incident asthma in males and females [10]. Asthma can either result from obesity (predominantly in women, with a later onset) or be worsened by obesity (i.e. poorer asthma control, with an earlier asthma onset, allergic type) [11]. The metabolic dysfunction of obesity may mediate this association [12]. Thus, recent data show a direct effect of high leptin levels, as encountered in obesity, on cytokine production, promoting allergic airway inflammation [13]. Moreover, in a recent longitudinal study, leptin mediated $>60 \%$ of the association between high body adiposity and persistent asthma over time [14].

Asthma and OSAS could be associated, especially in asthmatic patients with higher body mass index [15]. However, the mechanisms linking the two pathologies remain unclear. If the relationship between asthma severity and OSAS severity is variable, asthmatics exert worse sleep quality and daytime sleepiness, both symptoms of SDB. Clinical studies suggest that obesity is the link bridging together asthma and OSAS [16, 17]. Further studies are needed, focusing on a better definition of the phenotypes of both diseases.

Winfried Randerath (Solingen, Germany) introduced and summarised the latest conjoint statement of the European Academy of Neurology, European Sleep Research Society, European Stroke Organization and ERS Task force on the impact of sleep disorders on risk and outcome of stroke. SDB and stroke are intertwined pathologies [18]. Intermittent hypoxia, increased sympathetic activity and sleep fragmentation may play a role in this association. Thus, severe OSAS is recognised as a major risk for stroke [19]. CPAP may reduce stroke risk, especially in treatment compliant patients [20]. Following stroke, the prevalence of OSAS increases up to 50\% [21] and is associated with an increased stroke recurrence augmented mortality risk and a poorer prognosis for recovery [19, 22]. CPAP may improve stroke outcome [23]. Due to this strong relationship and based on the available evidence, OSAS must be actively diagnosed and treated after stroke [24].

Non-apnoea sleep-wake disorders may also be associated with an increased stroke risk and worse outcome. However, further research is needed to expand knowledge on the bidirectional relationship between sleep disorders and stroke.

Finally, Samuel Vergès (Grenoble, France) shed light on hypoxic conditioning as a new therapeutic modality. If hypoxia (sustained, intermittent, local or systemic) is a key pathophysiological mechanism in many diseases, it could also be protective as well. On the basis of epidemiological studies, individuals living at moderate altitude have a reduced prevalence of obesity, lung cancer and lower mortality from coronary heart disease and stroke $[25,26]$. Although still debated, elderly subjects with moderate OSAS appear to show lower mortality rates compared to healthy ones [27], suggesting that a small amount of hypoxia can be beneficial for our health status. Hypoxic conditioning refers to a therapeutic procedure by which a potentially deleterious stimulus (i.e. hypoxia) is applied near to but below the threshold of damage to the organism to prevent (pre-conditioning) or enhance healing (post-conditioning) [28]. Even supported by encouraging pre-clinical data $[28,29]$, the translation of hypoxic conditioning procedures to humans with pathological conditions remains to be pioneering. Some proof of the beneficial effects of 
hypoxic conditioning have been seen in COPD [30], in the reduction of metabolic risk markers [31], as well as in the improvement of function in spinal cord injury [32]. As there is a high inter-subject variability in the response to hypoxia, further research may address the question of the hypoxic dose and pattern, along with the identification of biomarkers of the beneficial response to hypoxic conditioning.

In conclusion, improvements in therapeutic strategies and care pathways of SDB and its associated comorbidities require an accurate appraisal of the pathophysiological mechanisms underlying their often bidirectional association. If intermittent hypoxia seems to play a major role in this deleterious association, it could also be protective in a dose-response relationship.

\section{Insights into the pathophysiology of SDB}

This oral session included eight studies about the pathophysiology of SDB and its treatment.

FITZPATRICK et al. [33] studied the effect of intermittent hypoxia on bone-marrow derived macrophages from mice in the presence or absence of lipopolysaccharide. They observed that intermittent hypoxia-induced polarisation of macrophages to M1 phenotype impeded insulin signalling, and activates interleukin-1 $\beta$ through Toll-like receptor-4. The effects of intermittent hypoxia were enhanced under lipopolysaccharide conditions. In this field, SACRAMENTO et al. [34] subjected rats to mild and severe intermittent hypoxia. After measuring fasting glycemia, insulinemia, C-peptide and insulin sensitivity, they found an increased release of insulin from the pancreas which was linked to the insulin resistance observed in response to mild and severe intermittent hypoxia.

However, disordered breathing and autonomic dysfunction are pathophysiological hallmarks of heart failure. Using a heart failure rat model where chemoreceptors of the retrotrapezoid nucleus (RTN) were destroyed, DíAz et al. [35] showed that episodic hypercapnia stimulation triggers ventilator plasticity and elicits cardiorespiratory abnormalities that depend on RTN neurons. Using the same model but eliminating the brainstem pre-sympathetic neurons (RVLM-C1) instead, Del Rio et al. [36] showed that they play a major role in development/maintenance of irregular breathing pattern.

In terms of cardiovascular consequences, Zou et al. [37] presented a study of the European Sleep Apnoea Database (ESADA). They analysed the polysomnography studies and arterial hypertension in a cohort of OSAS patients. They observed a potential mechanistic link between hypertension and severe cases of OSAS via an increased carbonic anhydrase activity.

This session also included a novel work focused on the potential relationship between OSA and lung cancer. MARHUENDA et al. [38] applied different hypoxia paradigms mimicking those occurring in OSAS, COPD and the combination of both, known as overlap syndrome. They concluded that the response of lung cancer cells to the hypoxic environment is extremely complex and hardly predictable, strongly depending on each cell type. It is important to highlight that this was awarded the best abstract in the field [38]. Other interesting study showed how intermittent hypoxia are transmitted to the fetus in a sleep apnoea ewe model. Almendros et al. [39] subjected pregnant ewes to intermittent hypoxia and measured oxygen partial pressure simultaneously on the femoral artery of the mother and in the umbilical vein. Finally, the work from Op DE BEECK et al. [40] showed that higher loop gain is associated with a reduced upper airway stimulation.

In summary, this session revealed novel mechanisms linking obstructive sleep apnoea (OSA) with some of its well-known metabolic, cardiovascular and cancer consequences which could provide new therapeutic targets in OSA.

\section{Current perspectives in SDB}

This poster discussion session included 18n studies providing novel insights on OSAS pathophysiology and how different treatments can improve its metabolic, neurocognitive and cardiovascular consequences.

Thiel et al. [41] completed a trial that included 70 OSAS patients under CPAP treatment for $>1$ year randomised to CPAP withdrawal for 2 weeks or not on neurometabolic and cardiometabolic markers. Liquid chromatography and mass spectrometry analyses from blood samples revealed a significant increase of 3,4-dihydroxyphenylglycol, norepinephrine and cortisol in those withdrawn from treatment. These results suggest that the elevated blood pressure in OSAS could be mediated through the sympathetic nervous system and hypothalamic-pituitary-adrenal axis activation [41]. ERNST et al. [42] prospectively studies OSAS patients with hypertension and found that the severity of OSAS in this population does not affect the prevalence of metabolic syndrome. Specifically, they did not observe differences in triglycerides, high-density lipoprotein cholesterol [42]. In contrast to these findings, two works presented from the ESADA showed improvements on metabolic variables in response to CPAP therapy. In the first work, Gunduz et al. [43] showed a significant decrease in cholesterol after CPAP treatment by including 1564 
OSAS subjects from the ESADA cohort [43]. In the second study, TAŞBAKAN et al. [44] reported a clinically relevant reduction of the glycated haemoglobin after CPAP therapy in 1608 patients. This improvement was stronger in type 2 diabetes mellitus and more severe OSAS patients and especially in those who lost more weight [44]. In addition to the metabolic syndrome, it has been well described that OSAS can affect the cognitive domains and gait control. In this field, BAILLIEul et al. [45] presented a randomised controlled study to ascertain whether CPAP therapy in severe OSAS patients can improve the gait control. They included a sham CPAP control group. To evaluate gait control, the authors performed a visuo-verbal cognitive task (Stroop test) which was completed by 21 subjects. After analysis, CPAP treatment was not able to improve gait control [45]. More studies are still needed in this field to understand the mechanisms involved and to prevent the damage caused by OSAS on cerebral functions.

Most of the data presented in the session was based on the use of CPAP for OSAS treatment. This device provides positive airway pressure to maintain the patency of the upper airway. Although technological improvements have increased CPAP compliance, LiISTRO et al. [46] presented preliminary results showing that the new generation CPAP machines increased air pressure during the inspiratory phase. They did not find any explanation to these findings [46]. In addition to CPAP, other potential ways of treatment were presented. RATNESWARAN et al. [47] presented results of a meta-analysis on the effectiveness of electrical stimulation of the upper airway. The authors pooled 20 clinical trials $(n=895)$ and analysed the effect of invasive hypoglossal nerve stimulation ( $\mathrm{n}=15$ trials) or noninvasive transcutaneous electrical stimulation ( $n=5$ trials) on the apnoea-hypopnoea index and the Epworth Sleepiness Scale. They found that both methodologies can reduce OSAS severity significantly improving its symptoms [47]. Finally, PéPIN et al. [48] have shown new data supporting the long-term efficacy and safety of solriamfetol (JZP-110), a selective dopamine and norepinephrine reuptake inhibitor, in treating excessive daytime sleepiness in narcolepsy and OSAS [48].

In summary, CPAP improves of the quality of life in OSA patients but its benefits on outcomes are not clear, at least in part, due to its poor compliance. Thus, the improvement of its technology and novel approaches for treating OSA such as the studies presented in this session are still needed.

\section{Current perspectives in OSA therapeutic plans and their effects}

The implementation of different OSAS therapeutic interventions and the validation of their efficacy in selected patients' subgroups are currently highly discussed topics. In this oral presentation, eight research groups presented some of their latest original research focusing on the therapeutic possibilities and the results of their implementation among patients with OSAS or other SDB. Three common research strands emerged: 1) the benefits of different positive airway pressure (PAP) therapies on cardiovascular and metabolic outcomes; 2) the role of and the factors influencing CPAP compliance; and 3) the impact of acetazolamide on high altitude-related severe nocturnal hypoxemia.

A latent class analysis among chronic heart failure (CHF) patients treated with adaptive servoventilation (ASV) enrolled in the FACE study [49] defined six phenogroups who significantly differed in time to first event, defined as death from any cause, unplanned hospitalisation for worsening heart failure, or cardiac transplant. The cluster that presented the worst prognosis encompassed patients with a left ventricular ejection fraction $(\mathrm{LVEF})<35 \%$, male sex, predominant central sleep apnoea, stroke and atrial fibrillation. Additional results from a retrospective analysis from the SERVE-HF study [50] showed no influence of the ASV device used on cardiovascular and non-cardiovascular death rates.

Furthermore, the effect of CPAP therapy on cardiovascular and metabolic events was analysed among patients with confirmed OSAS but insufficient symptoms to warrant CPAP therapy in the MOSAIC trial [51]. During a 2- to 5-year follow-up, it was observed that, although CPAP reduced daytime sleepiness, it failed to address cardiovascular or metabolic outcomes.

Interestingly, a 10-year follow-up showed a negative correlation between effective PAP adherence, particularly after 24 months, and cardiovascular and cerebrovascular events, especially when total PAP usage was at least 6 years. This in spite of an immediate apnoea-hypopnoea index drop after the onset of PAP therapy. Another group focused its research on patients with OSAS who were involved in a relationship, noting that retired couples showed the highest CPAP compliance. Furthermore, telemonitoring during the habituation phase of the CPAP therapy guaranteed a non-inferior CPAP adherence, according to a study that analysed comparable OSAS patients groups undergoing usual care or telemonitoring after a 1-year follow-up. Moreover, a post-hoc analysis of two randomised controlled trials on CPAP withdrawal suggested that CPAP withdrawal might influence dehydroepiandrosterone levels. The latter might contribute to the pathophysiology of erectile dysfunction and sexual dysfunction among patients with OSAS. 
Finally, a randomised controlled trial showed that a preventive treatment with acetazolamide reduced the incidence of severe nocturnal hypoxaemia and altitude-related periodic breathing among lowlanders with COPD sleeping for two nights at high altitude $(3100 \mathrm{~m})$.

In conclusion, the session highlighted that the effect of OSA therapies on cardiovascular and cerebrovascular prevention is still under discussion. Nevertheless, patient phenotyping and strategies for CPAP adherence optimisation, including telemonitoring, might lower cardiovascular mortality and even play a role in erectile and sexual dysfunction pathophysiology.

\section{Sleep and neuromuscular diseases: assessment and management of respiratory problems}

The symposium of sleep and neuromuscular diseases showcased recent research and new methods developed to optimise treatment in neuromuscular disease (NMD). Oscar H. Mayer (Pennsylvania, USA) shed light on respiratory pathophysiology of NMD and its impact on clinical progression. Respiratory muscle weakness as a manifestation of NMD, can lead to respiratory failure following a cascade of chest wall disorders and physiologic abnormalities [52]. Lung and chest wall compliance have been known to worsen in NMD resulting in increasing elastic load on respiratory muscles and lower lung volumes [53]. Indeed, lung stiffness can be caused by progressive loss of inspiratory and expiratory muscle function, decrease in vital capacity, progressive atelectasis, and alteration of tissue mechanics, whilst the elastic properties of the lungs are closely related to chest wall compliance [53, 54]. Pathophysiologic progression in NMD predisposes to progressive respiratory morbidity; however, there are some proactive interventions that can attenuate the deterioration [55, 56]. Four therapeutic interventions against the NMD consequences were demonstrated including airway clearance, mechanical ventilation support, stabilisation of the chest wall and spine, and lung volume recruitment manoeuvres.

Andrea Aliverti (Milan, Italy) provided an overview of the traditional and emerging methods for assessing ventilatory function in NMD. Respiratory pressure volume curves are significantly altered in NMD following reduced chest wall and lung compliance with decreased slope curves and generally lower operating volumes [57]. There are several methods and techniques that can be used to assess respiratory muscle function $[58,59]$. Briefly, the methods to assess respiratory muscles function can be grouped into invasive or noninvasive techniques; and split into the subgroups of volitional or non-volitional (requiring or not the patient's cooperation) [60]. There are known drawbacks for the majority of these methods but this is not the only that should be considered when assessing ventilatory function in NMD [60, 61]. For instance, in Duchenne muscular dystrophy, specific time-points of respiratory impairment are identified during disease progression, which should be considered for defining outcome measures in clinical trials and treatment strategies [62]. In regard to traditional methods for assessing ventilatory function in NMD, important spirometric variables including forced expiratory volume in $1 \mathrm{~s}\left(\mathrm{FEV}_{1}\right)$, forced vital capacity (FVC), total lung capacity (TLC), and peak expiratory flow were reported and the importance to refer to the predicted values was underlined. Several emerging methods were also mentioned including: 1) the sniff nasal inspiratory pressure as an index of global inspiratory muscle strength; 2) sleep evaluation; 3) spontaneous breathing during daytime; 4) ultrasound; 5) imaging (magnetic resonance imaging); and 6) optoelectronic plethysmography to assess the thoracoabdominal kinematics (i.e. the expansion of the different portion of the chest wall), which is very useful to noninvasively assess the use of the different respiratory muscles in NMD, and detect the asynchronous or paradoxical chest wall motion.

Michelle Chatwin (London, UK) spoke about the cough assist devices that are useful in patients with an ineffective cough due to muscle weakness in NMD. The optimal medical management in the NMD includes: 1) ensuring that reflux is treated; 2) evaluation for aspiration; 3) control of secretions; and 4) use of prophylactic inhaled antibiotics [63]. The role of cough assist devices can be very important for airway secretion clearance in NMD, when the peak of cough flow values are $<160 \mathrm{~L} \cdot \mathrm{min}^{-1}$ [64-66]. Setting cough assist devices involves adjustment of the positive and negative pressures, the inspiratory, expiratory and pause times, as well as the inspiratory flow rate [66-68]. Cough assist devices are more effective with greater exsufflation and longer insufflation times, whilst the optimisation of its settings in bulbar amyotrophic lateral sclerosis is critical [69-71].

Anita Simonds (London, UK) talked about the role of sleep and SDB in NMD including prognosis, therapeutic approach, and the impact and utilisation of health resources. SDB in NMD is caused by an exaggerated decline of lung volumes during supine sleep, a compromised physiologic adaptation to sleep, and specific disease features that may lead to upper airway collapse or heart failure [72]. Screening symptoms for nocturnal hypoventilation can be very subtle in NMD and the nocturnal oxygen saturation measured by pulse oximetry is not sufficient for the early detection of nocturnal hypoventilation. Therefore, the monitoring of nocturnal hypoventilation as part of the nocturnal respiratory assessments is recommended [73]. In relation to therapeutic approaches to nocturnal hypoventilation, noninvasive 
ventilation improves sleep-related symptoms and survival which can be even more prolonged with noninvasive ventilation settings optimisation $[74,75]$. Nevertheless, only one-third of adults with NMD receives respiratory specialist care at a frequency recommended by professional guidelines and this suggests an urgent need to improve the management of patients with NMD [76].

In summary, important advances have been made in NMD including therapeutic interventions to treat disease consequences, cough assist devices to support cough effectiveness, and several new methods for assessing ventilatory function. Recently, the nocturnal hypoventilation monitoring has been suggested as part of nocturnal respiratory assessment in NMD, whilst the overall provision of respiratory care needs to be increased for the majority of NMD patients.

\section{New insights into respiratory function testing}

In patients with COPD, dynamic hyperinflation and large intrathoracic pressure swings to overcome the increased elastic and resistive loads during exercise may have negative haemodynamic effects and thus contribute to exercise intolerance in those patients [77]. CHEYNe et al. [78], supported this mechanism by demonstrating that an artificial increase in dynamic hyperinflation and negative intrathoracic pressure by external inspiratory and expiratory resistance in healthy and young individuals, significantly reduced the left ventricle stroke volume and left ventricle end-diastolic volume (measured by echocardiography) during moderate intensity cycling exercise.

In the same area of interest, ALTER et al. [79], presented the results from 1591 patients with COPD (COSYCONET cohort). In that cohort, they found that a significant proportion of patients with suspicious echocardiographic findings were undiagnosed and untreated. A 36\% of patients who presented LVEF $<50 \%$ or left ventricle end-diastolic diameter $>56 \mathrm{~mm}$ had neither a cardiovascular history nor were they receiving a medical treatment. In addition, $38 \%$ of patients with LVEF $<50 \%$ and $53 \%$ with left ventricle end-diastolic diameter $>56 \mathrm{~mm}$ lacked both a cardiac diagnosis and medical treatment.

In another cohort study with 2665 subjects (COPDGene), ARJOMANDi et al. [80], examined the validity of a FRC/TLC ratio (a proxy of air trapping), measured by computed tomogrpahy imaging, to predict $\mathrm{FEV}_{1} / \mathrm{FVC}$ ratio decline over a period of 6 years in smokers with preserved spirometry. They showed that a higher FRC/TLC ratio was associated with a faster progression from non-spirometric to spirometric COPD. The study highlighted that smokers with higher FRC/TLC ratio as opposed to their counterparts with lower FRC/TLC ratio, exhibited a greater decline in $\mathrm{FEV}_{1}$ and a smaller decline in FVC that resulted in an overall differential decline in $\mathrm{FEV}_{1} / \mathrm{FVC}$ ratio over the examined period of 6 years.

In this context, the study by GonçALves et al. [81] aimed at identifying which patients best qualify for ambulatory oxygen through lung function tests. By including 315 patients with mild-to-moderate COPD, authors found that diffusing capacity of the lung for carbon monoxide was the best lung function test predictor for ambulatory oxygen indication. For diffusing capacity of the lung for carbon monoxide values $\leqslant 65 \%$, sensitivity and specificity analysis were $69 \%$ and $78 \%$, respectively, whilst other parameters of lung function tests such as $\mathrm{FEV}_{1}, \mathrm{FVC}$, TLC and transfer coefficient of the lung for carbon monoxide exhibited lower sensitivity and specificity values.

Two studies using home-monitoring technologies were presented by D'Cruz et al. [82] and ZimmermanN et al. [83]. D'CRUz et al. [82] investigated first whether measuring neural respiratory drive (NRD, parasternal electromyography) at home (for 30 days) is feasible and secondly whether NRD measurements can track clinical trajectory in patients with COPD $(n=10)$ after hospitalisation for an acute exacerbation of COPD (AECOPD). The study revealed that NRD measurements of parasternal muscles were feasible at home by demonstrating high adherence to home visits (188 (85\%) visits) and NRD measurements (373 (100\%) measures). Besides, the authors also found that changes in NRD tracers of the parasternal muscles were strongly associated with improvements in lung function parameters (i.e. inspiratory capacity and $\left.\mathrm{FEV}_{1}, \mathrm{r}^{2}>0.98-0.95\right)$ after hospital discharge for an AECOPD. ZIMMERMANN et al. [83] demonstrated that day-to-day variations in lung function measured at home by forced oscillation technique can reflect symptoms and quality of life of patients with COPD. The study showed that the variability of inspiratory reactance by forced oscillation technique in 16 patients with severe COPD was significantly related to both COPD Assessment Test and St Georges Respiratory Questionnaire scores measured for a period of seven consecutive days. In addition, changes in inspiratory reactance were sensitive enough to detect an AECOPD 3 days before the worsening of COPD symptoms.

In this framework, Petrocelli et al. [84] validated the surface mechanomyogram (sMMGdi) technique, for assessing lateral vibration of diaphragm fibres, with surface EMG measurement (sEMGdi) to relate to breathlessness (Borg scale) in 11 patients with COPD during different inspiratory threshold-loading intensities. The authors found moderate but significant correlations among sMMGdi and sEMGdi and 
Borg that ranged between 0.54-0.64 (Spearman) suggesting the potential value of sMMGdi measurements when investigating breathlessness in patients with COPD.

Finally, in a randomised, double-blinded crossover study, DomNik et al. [85], assessed overnight respiratory mechanics in 20 patients with moderate-to-severe COPD and 20 age-matched healthy individuals and determined how the abovementioned parameters could be affected by nocturnal long-acting bronchodilation (aclidinium bromide/formoterol fumarate). The authors demonstrated that overnight inspiratory capacity and $\mathrm{FEV}_{1}$ deteriorated progressively in patients with COPD compared to healthy subjects. Nocturnal administration of the bronchodilator in the patients reversed lung function decline, permitting equivalent ventilation with a less inspiratory neural drive. The latter findings were supported by the decrease in lung resistance and transdiaphragmatic pressure and improvements in EMGdi/tidal volume ratio following the administration of the bronchodilator compared to the placebo group.

In summary, the session supported previous evidence as well as provided new insights into the close interaction that exists between lung (i.e. respiratory function and mechanics of breathing) and heart function in patients with COPD. Furthermore, promising data with regards to the feasibility and the clinical importance of assessing respiratory muscle activity and lung function in patients with COPD within and beyond the hospital walls were presented.

\section{Exercise physiopathology in various respiratory diseases}

Exercise intolerance in respiratory diseases is characteristically multi-factorial [86], and in patients with COPD peripheral muscle abnormalities play an important role [87]. LouvarIs et al. [88] examined whether oxygen saturation distribution in different regions of the quadriceps muscle is different during exercise in COPD patients compared to controls. Near-infrared spectroscopy revealed greater oxygen saturation heterogeneity between exercising muscles, and lower oxygen delivery to the muscles for a standardised work rate in patients with COPD compared to controls. These preliminary results suggest that greater oxygen saturation heterogeneity between exercising muscles may indicate perturbations in muscle metabolism in COPD as a consequence of lower oxygen delivery.

Exertional dyspnoea is a cardinal feature of cardiac and respiratory diseases [89], but underlying mechanisms may differ between individual diseases. BERTON et al. [90], therefore, investigated the contribution of high chemostimulation versus abnormal lung mechanics to dyspnoea in patients with COPD and CHF, matched for peak aerobic capacity, during incremental cycling. Both groups of patients showed excessive ventilation compared to controls, however, dyspnoea as a function of work rate and ventilation was higher in COPD compared to $\mathrm{CHF}$ and controls. This was in line with a decline in inspiratory reserve volume in COPD; the so-called dynamic hyperinflation. This highlights that, while high chemostimulation has an important role in exertional dyspnoea in CHF, abnormalities in lung mechanics cause the greater symptom burden observed in patients with COPD with similar exercise capacity.

Hypoxaemia is often cited as an important determinant of dyspnoea in combined pulmonary fibrosis and emphysema (CPFE) [91]. To explore this assumption, NEDER et al. [92] contrasted responses to exercise in patients with CPFE versus pulmonary fibrosis matched for the severity of exertional hypoxaemia. In fact, excessive exertional ventilation (minute ventilation/carbon dioxide production nadir $>50$ ) and extensive emphysema (>15\%), but not hypoxaemia in particular, predicted poor exercise tolerance due to dyspnoea in CPFE. Therefore, hypoxaemia per se is not the main determinant of exertional dyspnoea in CPFE. Exacerbated chemostimulation owing to increased wasted ventilation and a downward displacement of the arterial carbon dioxide tension set-point holds a key mechanistic role [91].

In a cohort of long-term survivors of hematopoietic stem cell transplantation, MyrdaL et al. [93] sought to identify determinants of cardiorespiratory fitness using cardiopulmonary exercise testing, pulmonary function tests and echocardiography. Peak oxygen uptake was associated with usual physical activity (i.e. meeting World Health Organization recommendations), lung transfer factor for carbon monoxide and left ventricular systolic function in particular. The investigators concluded that modifiable lifestyle factors, as well as pulmonary and cardiac function, are important determinants of cardiopulmonary fitness in this patients.

Alleviating the unpleasant symptoms associated with exertion is a major challenge in respiratory diseases [94]. In a randomised crossover trial, Fernandes Pinto et al. [95] explored the effect that placement of elastic tape on the chest wall and abdomen had on thoracoabdominal mechanics, dyspnoea, exercise capacity and usual physical activity in patients with COPD. Thoracoabdominal asynchrony, ventilation and dyspnoea were reduced during constant-load cycling, and exercise tolerance increased during incremental cycling when elastic taping was used. The authors also reported a rise in moderate/ vigorous physical activity using this intervention. 
In patients with severe emphysema, DE ARAujo et al. [96] explored the effect of endobronchial valve placement on breathing pattern during constant work rate cycling. Following placement of the valve, ventilation was increased (with greater tidal volume and lower respiratory rate) while dyspnoea was reduced at isotime when compared to baseline. Interestingly, changes in endurance time correlated well with changes in dynamic inspiratory capacity. Patients with severe emphysema can, therefore, exercise longer with less dyspnoea after endobronchial valve placement as a consequence of improved breathing pattern and lung mechanics.

Excessive ventilation contributes to dyspnoea burden and exercise intolerance in mild COPD [97]. PHILIPS et al. [98] explored the effect of inhaled nitric oxide (NO) on ventilation, dyspnoea and exercise capacity in this patient population. Inhaled NO improved peak work rate and oxygen uptake in COPD compared to placebo but no change was observed in controls. Importantly, minute ventilation/carbon dioxide production and dyspnoea at standardised work rate dropped with the intervention in COPD only. Thus, inhaled NO improves exercise capacity in mild COPD through an attenuation of ventilation and breathlessness during exertion.

Impaired cerebral oxygenation may contribute to exercise intolerance [99], in particular in the face of extreme hypoxaemia [100, 101]. Exercise-related hypoxaemia is prevalent in patients with pulmonary arterial hypertension [102]. In a placebo-controlled trial, Boutou et al. [103] investigated the effect of oxygen supplementation on exercise duration, perceived exertion and cerebral oxygenation in pulmonary arterial hypertension patients. Supplemental oxygen, when compared to medical air, led to a greater increase in cerebral oxyhaemoglobin and a lesser increase in deoxyhaemoglobin, respectively, while exercise duration was prolonged. Therefore, in pulmonary arteria hypertension patients with exertional hypoxaemia, oxygen supplementation may enhance exercise tolerance through improved cerebral oxygenation.

Overall, this session provided novel insights into the underlying mechanisms of exercise limitation in diverse respiratory diseases and potential strategies to alleviate exertional symptoms and improve exercise tolerance in these patients.

Acknowledgements: We would like to thanks Isaac Almendros for his excellent role as an Assembly 4 Early Career Representative. This position is now being carried out by Andrea Crespo.

Conflict of interest: None declared.

Support statement: V. Andrianopoulos is the recipient of an ERS-EU RESPIRE2 Marie Sklodowska-Curie Postdoctoral Research Fellowship (MCF (8465)-2015).

\section{References}

1 Kaditis AG, Alonso Alvarez ML, Boudewyns A, et al. Obstructive sleep disordered breathing in 2- to 18-year-old children: diagnosis and management. Eur Respir J 2016; 47: 69-94.

2 Kaditis AG, Alonso Alvarez ML, Boudewyns A, et al. ERS statement on obstructive sleep disordered breathing in 1- to 23-month-old children. Eur Respir J 2017; 50: 1700985.

3 Hodges E, Marcus CL, Kim JY, et al. Depressive symptomatology in school-aged children with obstructive sleep apnea syndrome: incidence, demographic factors, and changes following a randomized controlled trial of adenotonsillectomy. Sleep 2018; 41: zsy180.

4 Galland B, Spruyt K, Dawes P, et al. Sleep disordered breathing and academic performance: a meta-analysis. Pediatrics 2015; 136: e934-e946.

5 Kheirandish-Gozal L, De Jong MR, Spruyt K, et al. Obstructive sleep apnoea is associated with impaired pictorial memory task acquisition and retention in children. Eur Respir J 2010; 36: 164-169.

6 Marcus CL, Moore RH, Rosen CL, et al. A randomized trial of adenotonsillectomy for childhood sleep apnea. N Engl J Med 2013; 368: 2366-2376.

7 Biggs SN, Vlahandonis A, Anderson V, et al. Long-term changes in neurocognition and behavior following treatment of sleep disordered breathing in school-aged children. Sleep 2014; 37: 77-84.

8 Marcus CL, Radcliffe J, Konstantinopoulou S, et al. Effects of positive airway pressure therapy on neurobehavioral outcomes in children with obstructive sleep apnea. Am J Respir Crit Care Med 2012; 185: 998-1003.

9 Joosten KF, Larramona H, Miano S, et al. How do we recognize the child with OSAS? Pediatr Pulmonol 2017; 52 260-271.

10 Beuther DA, Sutherland ER. Overweight, obesity, and incident asthma: a meta-analysis of prospective epidemiologic studies. Am J Respir Crit Care Med 2007; 175: 661-666.

11 Dixon AE, Poynter ME. Mechanisms of asthma in obesity. pleiotropic aspects of obesity produce distinct asthma phenotypes. Am J Respir Cell Mol Biol 2016; 54: 601-608.

12 Mohan A, Grace J, Wang BR, et al. The effects of obesity in asthma. Curr Allergy Asthma Rep 2019; 19: 49.

13 Zheng $\mathrm{H}, \mathrm{Wu} \mathrm{D}, \mathrm{Wu} \mathrm{X}$, et al. Leptin promotes allergic airway inflammation through targeting the unfolded protein response pathway. Sci Rep 2018; 8: 8905.

14 Li Z, Leynaert B, Dumas O, et al. Role of leptin in the association between body adiposity and persistent asthma: a longitudinal study. Obesity 2019; 27: 894-898. 
Kong DL, Qin Z, Shen H, et al. Association of obstructive sleep apnea with asthma: a meta-analysis. Sci Rep 2017; 7: 4088

Prasad B, Nyenhuis SM, Weaver TE. Obstructive sleep apnea and asthma: associations and treatment implications. Sleep Med Rev 2014; 18: 165-171.

Bonsignore MR, Pepin JL, Anttalainen U, et al. Clinical presentation of patients with suspected obstructive sleep apnea and self-reported physician-diagnosed asthma in the ESADA cohort. J Sleep Res 2018; 27: e12729.

Alexiev F, Brill AK, Ott SR, et al. Sleep-disordered breathing and stroke: chicken or egg? J Thorac Dis 2018; 10 : Suppl. 34, S4244-S4252.

Hermann DM, Bassetti CL. Role of sleep-disordered breathing and sleep-wake disturbances for stroke and stroke recovery. Neurology 2016; 87: 1407-1416.

McEvoy RD, Antic NA, Heeley E, et al. CPAP for prevention of cardiovascular events in obstructive sleep apnea. N Engl J Med 2016; 375: 919-931.

Seiler A, Camilo M, Korostovtseva L, et al. Prevalence of sleep-disordered breathing after stroke and TIA: a meta-analysis. Neurology 2019; 92: e648-e654.

Duss SB, Brill AK, Bargiotas P, et al. Sleep-wake disorders in stroke-increased stroke risk and deteriorated recovery? An evaluation on the necessity for prevention and treatment. Curr Neurol Neurosci Rep 2018; 18: 72. Brill AK, Horvath T, Seiler A, et al. CPAP as treatment of sleep apnea after stroke: A meta-analysis of randomized trials. Neurology 2018; 90: e1222-e1230.

Kernan WN, Ovbiagele B, Black HR, et al. Guidelines for the prevention of stroke in patients with stroke and transient ischemic attack: a guideline for healthcare professionals from the American Heart Association/ American Stroke Association. Stroke 2014; 45: 2160-2236.

Faeh D, Gutzwiller F, Bopp M. Lower mortality from coronary heart disease and stroke at higher altitudes in Switzerland. Circulation 2009; 120: 495-501.

Faeh D, Moser A, Panczak R, et al. Independent at heart: persistent association of altitude with ischaemic heart disease mortality after consideration of climate, topography and built environment. J Epidemiol Community Health 2016; 70: 798-806.

Lavie P, Lavie L. Unexpected survival advantage in elderly people with moderate sleep apnoea. J Sleep Res 2009; 18: $397-403$.

Verges S, Chacaroun S, Godin-Ribuot D, et al. Hypoxic conditioning as a new therapeutic modality. Front Pediatr 2015; 3: 58

Baillieul S, Chacaroun S, Doutreleau S, et al. Hypoxic conditioning and the central nervous system: A new therapeutic opportunity for brain and spinal cord injuries? Exp Biol Med (Maywood) 2017; 242: 1198-1206.

Burtscher M, Haider T, Domej W, et al. Intermittent hypoxia increases exercise tolerance in patients at risk for or with mild COPD. Respir Physiol Neurobiol 2009; 165: 97-103.

Haufe S, Wiesner S, Engeli S, et al. Influences of normobaric hypoxia training on metabolic risk markers in human subjects. Med Sci Sports Exerc 2008; 40: 1939-1944.

Hayes HB, Jayaraman A, Herrmann M, et al. Daily intermittent hypoxia enhances walking after chronic spina cord injury: a randomized trial. Neurology 2014; 82: 104-113.

Fitzpatrick S, Mcgillicuddy F, Roche H, et al. Intermittent hypoxia activates the Interleukin (IL)-1ß and nuclea factor (NF)KB signaling pathway in primary murine macrophages. Eur Respir J 2019; 54: Suppl. 63, OA4932.

Sacramento JF, Melo BF, Olea E, et al. Hyperinsulinemia due to altered insulin secretion contributes to insulin resistance in chronic intermittent hypoxia independently of obesity. Eur Respir J 2019; 54: Suppl. 63, OA4935.

Díaz HS, Andrade DC, Toledo C, et al. Episodic stimulation of central chemoreceptor neurons elicits disordered breathing and autonomic dysfunction in volume overload heart failure. Am J Physiol Cell Mol Physiol 2020; 318: L27-L40.

Del Rio R, Andrade DC, Toledo C, et al. Carotid body-mediated chemoreflex drive in the setting of low and high output heart failure. Sci Rep 2017; 7: 8035.

Zou D, Grote L, Basoglu OK, et al. Increased arterial standard bicarbonate is associated with hypertension in patients with obstructive sleep apnea - data from the European Sleep Apnea DatAbase (ESADA). Eur Respir J 2019; 54: Suppl. 63, OA4933.

Marhuenda E, Campillo N, Gabasa M, et al. Effects of sustained and intermittent hypoxia on human lung cancer cells. Am J Respir Cell Mol Biol 2019; 61: 540-544.

Almendros I, Martínez-Ros P, Farré N, et al. Placental oxygen transfer reduces hypoxia-reoxygenation swings in fetal blood in a sheep model of gestational sleep apnea. J Appl Physiol 2019; 127: 745-752.

Op de Beeck S, Wellman A, Strohl K, et al. Loop gain as a determinant of upper airway stimulation efficacy in sleep apnoea. Eur Respir J 2019; 54: Suppl. 63, OA4934.

Thiel S, Haile SR, Peitzsch M, et al. Endocrine responses during CPAP-withdrawal in obstructive sleep apnea: data from two randomised controlled trials. Eur Respir J 2019; 54: Suppl. 63, PA1996.

apnea and hypertension. Eur Respir J 2019; 54: Suppl. 63, PA2010.

Gunduz C, Basoglu OK, Schiza S, et al. The effect of positive airway pressure on cholesterol in patients with sleep apnea: data from the European Sleep Apnea Network (ESADA). Eur Respir J 2019; 54: Suppl. 63, PA2005.

Taşbakan MS, Grote L, Hedner J, et al. Positive airway pressure treatment reduces glycated hemoglobin (HbA1c) levels in obstructive sleep apnea patients: longitudinal data from the ESADA. Eur Respir J 2019; 54: Suppl. 63, PA2007.

5 Baillieul S, Wuyam B, Tamisier R, et al. Impaired control of gait in severe obstructive sleep apnea and continuous positive airway pressure treatment: a randomized controlled trial. Eur Respir J 2019; 54: Suppl. 63, PA1997.

6 Liistro G, Dury M, Chantrain VA, et al. Unexpected large pressure increases during inspiration with new generation CPAP devices. Eur Respir J 2019; 54: Suppl. 63, PA2002.

Ratneswaran C, Guni A, Al-Sherif M, et al. Electrical stimulation as treatment for obstructive sleep apnoea: a meta-analysis. Eur Respir J 2019; 54: Suppl. 63, PA1994.

Pépin JL, Schwab R, Shapiro C, et al. Long-term efficacy of solriamfetol for excessive sleepiness in narcolepsy or obstructive sleep apnea. Eur Respir J 2019; 54: Suppl. 63, PA1998. 
D'Ortho MP, Tamisier R, Levy P, et al. FACE: A prospective multicentre observational cohort study in patients with chronic heart failure (CHF) and central sleep apnoea (CSA) treated with adaptive servoventilation (ASV). Eur Respir J 2013; 42: Suppl. 57, P324.

50 Cowie MR, Woehrle H, Wegscheider $\mathrm{K}$, et al. Rationale and design of the SERVE-HF study: treatment of sleep-disordered breathing with predominant central sleep apnoea with adaptive servo-ventilation in patients with chronic heart failure. Eur J Heart Fail 2013; 15: 937-943.

51 Turnbull CD, Craig SE, Kohler M, et al. Cardiovascular event rates in the MOSAIC trial: 2-year follow-up data. Thorax 2014; 69: 950.

52 Mehta S. Neuromuscular disease causing acute respiratory failure. Respir Care 2006; 51: 1016-1021.

53 Benditt JO, Boitano LJ. Pulmonary issues in patients with chronic neuromuscular disease. Am J Respir Crit Care Med 2013; 187: 1046-1055.

54 Shahrizaila N, Kinnear WJ, Wills AJ. Respiratory involvement in inherited primary muscle conditions. J Neuro Neurosurg Psychiatry 2006; 7: 1108-1115.

55 Pfeffer G, Povitz M. Respiratory management of patients with neuromuscular disease: current perspectives Degener Neurol Neuromuscul Dis 2016; 6: 111-118. Ambrosino N, Carpene N, Gherardi M. Chronic respiratory care for neuromuscular diseases in adults. Eur Respir J 2009; 34: 444-451.

57 Lo Mauro A, Aliverti A. Physiology of respiratory disturbances in muscular dystrophies. Breathe 2016; 12: 318-327.

58 Laveneziana P, Albuquerque A, Aliverti A, et al. ERS statement on respiratory muscle testing at rest and during exercise. Eur Respir J 2019; 53: 1801214

59 Oliveira MJP, Rodrigues F, Firmino-Machado J, et al. Assessment of respiratory muscle weakness in subjects with neuromuscular disease. Respir Care 2018; 63: 1223-1230.

60 Caruso P, Albuquerque AL, Santana PV, et al. Diagnostic methods to assess inspiratory and expiratory muscle strength. J Bras Pneumol 2015; 41: 110-123.

61 Steier J, Kaul S, Seymour J, et al. The value of multiple tests of respiratory muscle strength. Thorax 2007; 62: 975-980.

62 LoMauro A, Romei M, Gandossini S, et al. Evolution of respiratory function in Duchenne muscular dystrophy from childhood to adulthood. Eur Respir J 2018; 51: 1701418

63 Eckerland M, Bock C, Olivier M, et al. Reducing the frequency of respiratory tract infections in severe neurological disorders by inhaled antibiotics: a retrospective data analysis. ERJ Open Res 2019; 5: 00149-2018.

64 Sancho J, Servera E, Diaz J, et al. Predictors of ineffective cough during a chest infection in patients with stable amyotrophic lateral sclerosis. Am J Respir Crit Care Med 2007; 175: 1266-1271.

65 Toussaint M, Chatwin M, Gonzales J, et al. 228th ENMC International Workshop: Airway clearance techniques in neuromuscular disorders Naarden, The Netherlands, 3-5 March, 2017. Neuromuscul Disord 2018; 28: 289-298.

66 Homnick DN. Mechanical insufflation-exsufflation for airway mucus clearance. Respir Care 2007; 52 : $1296-1305$.

67 Chatwin M, Toussaint M, Goncalves MR, et al. Airway clearance techniques in neuromuscular disorders: a state of the art review. Respir Med 2018; 136: 98-110.

68 Chatwin M, Simonds AK. Long-term mechanical insufflation-exsufflation cough assistance in neuromuscular disease: patterns of use and lessons for application. Respir Care 2020; 65: 135-143.

69 Bach JR, Mahajan K, Lipa B, et al. Lung insufflation capacity in neuromuscular disease. Am J Phys Med Rehabil 2008; 87: 720-725.

70 Andersen T, Sandnes A, Brekka AK, et al. Laryngeal response patterns influence the efficacy of mechanical assisted cough in amyotrophic lateral sclerosis. Thorax 2017; 72: 221-229.

71 Volpe MS, Naves JM, Ribeiro GG, et al. Airway clearance with an optimized mechanical insufflation-exsufflation maneuver. Respir Care 2018; 63: 1214-1222.

72 Aboussouan LS. Sleep-disordered breathing in neuromuscular disease. Am J Respir Crit Care Med 2015; 191: 979-989.

73 Trucco F, Pedemonte M, Fiorillo C, et al. Detection of early nocturnal hypoventilation in neuromuscular disorders. J Int Med Res 2018; 46: 1153-1161.

74 Morelot-Panzini C, Bruneteau G, Gonzalez-Bermejo J. NIV in amyotrophic lateral sclerosis: the 'when' and 'how' of the matter. Respirology 2019; 24: 521-530.

75 Bourke SC, Tomlinson M, Williams TL, et al. Effects of non-invasive ventilation on survival and quality of life in patients with amyotrophic lateral sclerosis: a randomised controlled trial. Lancet Neurol 2006; 5: 140-147.

76 Rose L, McKim D, Leasa D, et al. Patterns of healthcare utilisation for respiratory complications of adults with neuromuscular disease: a population study. Eur Respir J 2018; 52: 1800754.

77 Vogiatzis I, Zakynthinos S. Factors limiting exercise tolerance in chronic lung diseases. Comp Physiol 2012; 2: 1779-1817.

78 Cheyne W, Watson A, Welykholowa $\mathrm{K}$, et al. The effect of negative intrathoracic pressure and dynamic hyperinflation on heart-lung interaction during exercise. Eur Respir J 2019; 54: Suppl. 63, OA474.

79 Alter P, Mayerhofer B, Kahnert K, et al. Influences of lung and left heart function on respiratory symptoms in COPD: results from the COSYCONET cohort. Eur Respir J 2019; 54: Suppl. 63, OA479.

80 Arjomandi M, Zeng S, Lynch D, et al. Patterns of development of airflow limitation in smokers with preserved spirometry and air trapping phenotype in COPDGene. Eur Respir J 2019; 54: Suppl. 63, OA476.

81 Gonçalves G, Cabral S, Lopes AP. Predicting ambulatory oxygen indications with lung function tests. Eur Respir J 2019; 54: Suppl. 63, OA480.

82 D'Cruz R, Suh E, Kaltsakas G, et al. Feasibility of measuring neural respiratory drive in the home following severe COPD exacerbation. Eur Respir J 2019; 4: Suppl. 63, OA475.

83 Zimmermann S, Huvanandana J, Nguyen C, et al. Temporal variability of forced oscillometry from home telemonitoring and relationship with patient-centred outcomes and AECOPD. Eur Respir J 2019; 54: Suppl. 63, OA477.

84 Petrocelli E, Lozano-García M, Moore A, et al. The relationship between the diaphragm mechanomyogram and breathlessness during inspiratory loading in chronic obstructive pulmonary disease. Eur Respir J 2019; 54: Suppl. 63, OA481. 
Domnik N, James M, Ciavaglia C, et al. Overnight deterioration of supine respiratory mechanics in COPD: impact of nocturnal aclidinium bromide/formoterol fumarate. Eur Respir J 2019; 54: Suppl. 63, OA478.

Neder JA, Marillier M, Bernard AC, et al. The integrative physiology of exercise training in patients with COPD. COPD 2019; 16: 182-195.

Maltais F, Decramer M, Casaburi R, et al. An official American Thoracic Society/European Respiratory Society statement: update on limb muscle dysfunction in chronic obstructive pulmonary disease. Am J Respir Crit Care Med 2014; 189: e15-e62.

Louvaris Z, Rodrigues A, Dierckx S, et al. Heterogeneity of metabolism and activation in lower limb muscles during exercise in COPD: a preliminary data analysis. Eur Respir J 2019; 54: Suppl. 63, OA3565.

Neder JA, Berton DC, Marillier M, et al. The role of evaluating inspiratory constraints and ventilatory inefficiency in the investigation of dyspnea of unclear etiology. Respir Med 2019; 158: 6-13.

Berton DC, Balzan F, Plachi F, et al. Mechanisms of exertional dyspnoea in COPD and heart failure patients matched by peak aerobic capacity. Eur Respir J 2019; 54: Suppl. 63, OA3567.

Costa CM, Neder JA, Verrastro CG, et al. Uncovering the mechanisms of exertional dyspnoea in combined pulmonary fibrosis and emphysema. Eur Respir J 2019: 1901319.

Neder JA, Costa C, Verrastro C, et al. Mechanisms and consequences of exertional dyspnoea in combined pulmonary fibrosis and emphysema (CPFE). Eur Respir J 2019; 54: Suppl. 63, OA3569.

Myrdal OH, Diep PP, Ruud E, et al. Determinants of cardiorespiratory fitness in young long-term survivors of hematopoietic stem cell transplantation (HSCT). Eur Respir J 2019; 54: Suppl. 63, OA3568.

Spruit MA, Singh SJ, Garvey C, et al. An Official American Thoracic Society/European Respiratory Society Statement: Key Concepts and Advances in Pulmonary Rehabilitation. Am J Respir Crit Care Med 2013; 188: e13-e64.

Fernandes Pinto T, Fagundes Xavier R, Lunardi A, et al. Effects of the elastic tape on thoracoabdominal mechanic, dyspnea, exercise capacity and physical activity level in patients with COPD. Eur Respir J 2019; 54 Suppl. 63, OA3566.

De Araujo CL, Blondeel A, Rodrigues A, et al. Endobronchial valve placement impact on breathing pattern during exercise in patients with severe emphysema. Eur Respir J 2019; 54: Suppl. 63, OA3570. breathing reserve in the assessment of exertional dyspnea in COPD. COPD 2019; 16: 174-181.

Philips D, Brotto A, Ross B, et al. The effect of inhaled nitric oxide on dyspnea and exercise capacity in mild COPD. Eur Respir J 2019; 54: Suppl. 63, OA3572.

Marillier M, Gruet M, Baillieul S, et al. Impaired cerebral oxygenation and exercise tolerance in patients with severe obstructive sleep apnea syndrome. Sleep Med 2018; 51: 37-46.

Verges S, Rupp T, Jubeau M, et al. Cerebral perturbations during exercise in hypoxia. Am J Physiol Regul Integr Comp Physiol 2012; 302: R903-R916.

Marillier M, Arnal PJ, Le Roux Mallouf T, et al. Effects of high-altitude exposure on supraspinal fatigue and corticospinal excitability and inhibition. Eur J Appl Physiol 2017; 117: 1747-1761.

Righini FM, Apostolo A, Heck PB, et al. Exercise physiology in pulmonary hypertension patients with and without congenital heart disease. Eur J Prev Cardiol 2019; 26: 86-93.

Boutou A, Dipla K, Zafeiridis A, et al. Acute effects of oxygen supplementation during exercise in patients with pulmonary arterial hypertension. Eur Respir J 2019; 54: Suppl. 63. OA3571. 